\title{
Peripheral Neuropathies Associated With Diabetes Mellitus: A Review
}

\author{
Abu Khalid Muhammad Maruf Raza
}

\begin{abstract}
The review aimed to describe various diabetes mellitus-associated peripheral neuropathies, various related diseases and clinical features. English-language literature search using a combination of words (diabetic neuropathy and diagnosis) was used to identify original studies, consensus statements, and reviews published in the last few years. The diverse neuropathies of diabetes mellitus can be seen in various research articles which are found in clinical practice. Prompt diagnosis and recognition of these with the institution of appropriate treatment measures would go a long way towards reducing morbidity and mortality outcomes.
\end{abstract}

Keywords: Diabetes; Peripheral; Neuropathies

\section{Introduction}

Diabetic peripheral neuropathy is defined as the presence of symptoms and signs of peripheral nerve dysfunction in people with diabetes after the exclusion of other causes [1]. It manifests in the somatic, sensory and/or autonomic parts of the peripheral nervous system [2]. The sensory phenotype is divided into small, large or mixed fiber types. Symptoms of neuropathy are very common, and subclinical neuropathy is more common than clinical neuropathy [3]. Neuropathy may remain undetected, and progress over time leading to serious complications. With the rising global burden of diabetes, peripheral neuropathy, and other diabetes complications are expected to be on the increase. This would negatively affect their quality of life and mortality. The most common clinical presentation of diabetic peripheral neuropathy is distal symmetrical polyneuropathy [4].

This review focuses on the common neuropathies encountered in clinical practice. We also bring to fore the rarely thought about neuropathies commonly misdiagnosed as other conditions. A good knowledge of these neuropathies would go

Manuscript accepted for publication December 07, 2016

Department of Pathology, Jahurul Islam Medical College, Bajitpur, Kishoregonj, Bangladesh. Email: drmarufraza@gmail.com

doi: https://doi.org/10.14740/jnr412e a long way in improving the patient care offered by the diabetes clinician.

\section{Anatomic Considerations}

Small fiber neuropathies manifest with painful paresthesias most commonly over the lower limb. The pain may be dull, aching, burning, lancinating or cramp-like. Paresthesias may manifest as a sensation of coldness, numbness or tingling. There may be associated diminution of pain and temperature perception in the lower limbs in a glove and stocking distribution. Features of large fiber neuropathy include loss of ankle jerk, impaired position, and vibration sensory ataxia. Mixed small and large fiber neuropathy is the most common variety of painful diabetic neuropathy [5].

\section{Acute Painful Neuropathy}

First described by Archer and Watkins [6] in 1983, is a distinct and common variant of distal symmetrical polyneuropathy that presents with abrupt onset of severe sensory symptoms with little or no sensory and motor signs. It usually follows a period of change in glycemic control. It usually starts with rapid weight loss over a short period followed by severe, unremitting pain mostly in the feet. Optimizing glycemic control eventually leads to weight gain and remission of symptoms [7].

\section{Chronic Sensorimotor Distal Symmetrical Poly- neuropathy (DSPN)}

Chronic sensorimotor DSPN is the most common form of diabetic neuropathy. It is present in more than $10 \%$ of patients at the diagnosis of type 2 diabetes with an insidious onset. More than $80 \%$ of patients with clinical diabetic neuropathy have a distal symmetrical form of the disorder [8].

Symptoms may be positive or negative. Positive symptoms include feelings of pins and needles, tingling, burning, and neuropathic pain. Negative symptoms include numbness, impaired tactile, thermal and pain sensation. Positive symptoms, probably due to neural hyperexcitability, include pins and needles and pain which may be of varying qualities (burning, aching or lancinating). These negative and positive sen- 
sory symptoms may coexist. Symptoms begin distally in the toes and the feet and gradually extend proximally to involve the hands and fingers. This pattern of spread or progression reflects the dying-back nature of underlying nerve damage [9].

\section{Painful Small Fiber Neuropathy}

It is a variant of distal sensorimotor polyneuropathy in which the small myelinated fibers are affected alone or out of proportion to large nerve fibers. Key complaints are burning or stabbing pain in the feet which may be spontaneous [10]. This form of neuropathy is usually distressing and debilitating, impairing patient's quality of life.

\section{Focal Limb Neuropathies}

Most persons with diabetes and upper limb neuropathic symptoms and signs will either have a mononeuropathy or multiple mononeuropathies. This adds to the disability already imposed by the polyneuropathy that is almost always present. Ulnar neuropathies in people with diabetes are often insidious and are mainly motor with limited sensory symptoms and signs. Such focal neuropathies can easily go undetected because their symptoms are thought to be due to a polyneuropathy [11]. When sensory or motor symptoms are more prominent in the hands than feet, carpal tunnel syndromes or ulnar neuropathies should be suspected and excluded.

\section{Cranial Neuropathies (Diabetic Ophthalmople- gia)}

Oculomotor nerve palsies are the most common cranial neuropathy observed in diabetic patients. It rarely occurs in children. It affects mostly middle-aged adults. The pupillary function is spared. It has been attributed to ischemia occurring centrally within the third nerve, preserving the peripherally located parasympathetic pupil-constrictor fibers. This is in contrast to compressive lesions of the oculomotor nerve, such as an aneurysm of the posterior communicating artery, in which the pupillary fibers are affected [12]. Sixth nerve palsies also occur, but rarely. It is unclear whether seventh nerve palsies occur more frequently in people with diabetes than in the general population.

\section{Compression Neuropathies}

Compression or entrapment neuropathies are more common in people with diabetes. They include carpal tunnel syndrome (CTS), ulnar neuropathy at the elbow (UNE), meralgia paraesthetica (entrapment of the lateral femoral cutaneous nerve of the thigh) at the inguinal ligament or peroneal neuropathy at the fibular head [13]. Nerve conduction studies should be carried to conform compressive median or ulnar mononeuropathies and to screen for associated axonal injury. Those found to have pure demyelinating neuropathy usually respond well to positional splints while those with active demyelination are treated with carpal tunnel decompression which is nearly as effective for diabetic patients as for normoglycemic controls [14].

\section{Diabetic Truncal Radiculoneuropathy}

It occurs in the setting of long-standing diabetes with other complications, especially polyneuropathy. Most of the affected individuals are in the fifth or sixth decade of life with a variable duration of diabetes [15]. It presents gradually with painful paresthesias in variable size patches unilaterally or bilaterally in the lower anterior chest or upper abdomen with nocturnal worsening. Associated involvement of motor nerve fibers can lead to bulging of the abdominal wall in the paraesthetic areas, best appreciated when the patient is standing.

\section{Chronic Inflammatory Demyelinating Polyneu- ropathy (CIDP)}

Patients with diabetes mellitus (DM) seem to develop clinical and electrophysiologic characteristics in keeping with CIDP. This condition tends to occur more frequently in people with diabetes than in non-diabetics. The rapid onset and progression of the neuropathy, demyelinating features on nerve conduction studies and an excellent response to immunomodulatory treatments distinguish this entity from the far more frequent chronic diabetic sensorimotor polyneuropathy [16]. Treatment should be instituted promptly to prevent ongoing demyelination and the secondary axonal loss that would result in permanent disability.

\section{Hypoglycemic Neuropathy}

It is a distal symmetrical predominantly sensory neuropathy occurring on a background of recurrent episodic symptoms secondary to hypoglycemia. Hypoglycemia causes effects in both the central and peripheral nervous systems. Energy depletion plays a key role in the pathogenesis of hypoglycemiainduced neuropathy [17]. Ischemia also plays a role. The electrophysiological findings are suggestive of a primary axonal neuropathy with evidence of secondary demyelination. The pathologic changes in hypoglycemic neuropathy may include axonal neuropathy, anterior horn cells destruction in cervical spinal cord with normal dorsal and ventral roots and dorsal root ganglia, or even a normal nerve [18]. There is a need to exclude the presence of an insulinoma as the literature on humans developing a hypoglycemic neuropathy is small and related to the presence of an insulinoma.

\section{Impaired Glucose Tolerance Neuropathy}

This occurs in persons with normal fasting glucose and glycosylated hemoglobin values. However, they have impaired 
glucose tolerance (IGT) on oral glucose tolerance testing. This form of diabetic neuropathy manifests as a predominantly sensory neuropathy. Small fiber neuropathic changes occur more commonly in persons with IGT than the normal population [19]. This neuropathy is clinically similar to early diabetic neuropathy with a predilection for small fiber damage leading to distressing pain and autonomic symptoms.

\section{Diabetic Autonomic Neuropathy (DAN)}

Autonomic nerve involvement is probably the most undiagnosed complication. DAN may present in multiple organ systems in undiagnosed patients and can result in significant morbidity and mortality. Autonomic dysfunction may already exist at the time of type 2 DM diagnosis, and its prevalence in the diabetic population rises with time. In type $1 \mathrm{DM}$, hypoglycemia unawareness is the most common symptom [20]. Autonomic nerve fibers are invariably involved in chronic sensorimotor polyneuropathy, frequently subclinical in the early stages of the polyneuropathy, although it may be detected using sensitive methods to measure and quantify autonomic function. When symptomatic, this may result in impaired sweating and some skin vasomotor changes. However, the autonomic nervous system may become widely involved and dominate the clinical picture. In most patients, the symptoms are not severe, but some have devastating DAN. The neuropathy may affect all or selected organs or systems innervated by the autonomic nervous system. Thus one or more of the following may develop gastroparesis, diarrhea, constipation, orthostatic hypotension, bladder dysfunction, and erectile dysfunction. About $40 \%$ of diabetic men develop erectile dysfunction which may occur in the absence of, or in association with, other manifestations of DAN [21]. The clinical examination of the autonomic nervous system is limited. A resting tachycardia and a fixed heart rate of deep breathing or when the patient goes from lying to standing indicate vagal parasympathetic dysfunction. The simple bedside measurement of lying-standing blood pressure change is an important test for sympathetic vasoconstrictor dysfunction.

\section{Conclusion}

It is important for clinicians to be knowledgeable about the various neuropathic complications of DM. Autonomic involvement which is a leading cause of mortality in these patients may mistake for other disease complications. Prompt diagnosis and recognition of these with the institution of appropriate treatment measures would go a long way towards reducing morbidity and mortality outcomes.

\section{References}

1. Boulton AJ, Malik RA, Arezzo JC, Sosenko JM. Diabetic somatic neuropathies. Diabetes Care. 2004;27(6):14581486.
2. Boulton AJ, Vinik AI, Arezzo JC, Bril V, Feldman EL, Freeman R, Malik RA, et al. Diabetic neuropathies: a statement by the American Diabetes Association. Diabetes Care. 2005;28(4):956-962.

3. Eastman RC. Neuropathy in Diabetes. In: Harris MI, Ed. Diabetes in America, National Diabetes Information, Clearinghouse, 2nd Edition. 1995;339-360.

4. Kasznicki J. Advances in the diagnosis and management of diabetic distal symmetric polyneuropathy. Arch Med Sci. 2014;10(2):345-354.

5. Vinik AI, Mehrabyan A. Diabetic neuropathies. Med Clin North Am. 2004;88(4):947-999, xi.

6. Archer AG, Watkins PJ, Thomas PK, Sharma AK, Payan J. The natural history of acute painful neuropathy in diabetes mellitus. J Neurol Neurosurg Psychiatry. 1983;46(6):491-499.

7. Thomas PK. Classification, differential diagnosis, and staging of diabetic peripheral neuropathy. Diabetes. 1997;46(Suppl 2):S54-57.

8. De Freitas MRG. Diabetic neuropathy I-Epidemiology, classification, clinical and electrophysiologic aspects. A study of 210 cases. Rev Brasileira Neurol. 1992;28:6973.

9. Boulton AJ, Armstrong WD, Scarpello JH, Ward JD. The natural history of painful diabetic neuropathy - a 4-year study. Postgrad Med J. 1983;59(695):556-559.

10. Vital C, Vital A, Dupont M, Gin H, Rouanet-Larriviere M, Lacut JY. Acute painful diabetic neuropathy: two patients with recent IDDM. Journal of the Peripheral Nervous System. 1996;2(2):151-154.

11. Dyck PJ, Dyck PJB, Englestad J. Pathologic alterations of nerve. Peripheral Neuropathy. 2005;1:733-829.

12. Said G. Focal and multifocal diabetic neuropathies. Arq Neuropsiquiatr. 2007;65(4B):1272-1278.

13. Asbury AK, Aldredge H, Hershberg R, Fisher CM. Oculomotor palsy in diabetes mellitus: a clinico-pathological study. Brain. 1970;93(3):555-566.

14. Thomsen NO, Cederlund R, Rosen I, Bjork J, Dahlin LB. Clinical outcomes of surgical release among diabetic patients with carpal tunnel syndrome: prospective follow-up with matched controls. J Hand Surg Am. 2009;34(7):1177-1187.

15. Pourmand R. Diabetic neuropathy. Neurol Clin. 1997;15(3):569-576.

16. Krendel DA, Costigan DA, Hopkins LC. Successful treatment of neuropathies in patients with diabetes mellitus. Arch Neurol. 1995;52(11):1053-1061.

17. Hsu YC, Zhan HL, Yang CP. Clinical and experimental evidence of hypoglycemic neuropathy. Diabetes Res Open Journal. 2015;1(5):131-135.

18. Tom MI, Richardson JC. Hypoglycemia from islet cell tumour of pancreas with amyotrophy and cerebrospinal nerve cell changes: A case report. Journal of Neuropathology and Experimental Neurology. 1951;10(1):57-66.

19. Boulton AJ, Malik RA. Neuropathy of impaired glucose tolerance and its measurement. Diabetes Care. 2010;33(1):207-209.

20. Vinik AI, Maser RE, Mitchell BD, Freeman R. Diabetic autonomic neuropathy. Diabetes Care. 2003;26(5):1553- 
1579.

21. Toyry JP, Niskanen LK, Mantysaari MJ, Lansimies EA, Uusitupa MI. Occurrence, predictors, and clinical sig- nificance of autonomic neuropathy in NIDDM. Ten-year follow-up from the diagnosis. Diabetes. 1996;45(3):308315. 